\title{
MS061.P02
}

\section{Crystal symmetry-magnetism correlations in Ruthenate double perovskites}

\author{
$\underline{\text { Sean Dusan Injac }}^{1}$, Paula Kayser-Gonzales ${ }^{2}$, Brendan Kennedy ${ }^{1}$ \\ ${ }^{1}$ School Of Chemistry, University Of /sydney, Sydney, Australia, ${ }^{2}$ Instituto de Ciencia de Materiales de Madrid, C.S.I.C., \\ Cantoblanco, E-28049, Madrid, Spain \\ E-mail: ssta8177@uni.sydney.edu.au
}

Ruthenate perovskite oxides of the form A2BRuO6 have been found to show unusual magnetic ground states, often showing competing ferromagnetic (FM) and antiferromagnetic (AFM) interactions. Of note is the double perovskite Sr2YRuO6 which shows unusual magnetic response at low temperatures, as well as triple state superconductivity when $10 \%$ substitution of Ru for Cu occurs.[1][2] Previous studies have investigated the series Ba2-xSrxYRuO6 in order to probe a possible relationship between crystal structure and these unusual magnetic properties.[3] Furthering this research the isostructral series Ba2-xSrxHoRuO6 was synthesised. The compounds show the following structural progression throughout the series: Fm-3m $(0 \leq x \leq 0.6) \rightarrow \mathrm{I} / \mathrm{m}(\mathrm{x}=0.8) \rightarrow \mathrm{I} 2 / \mathrm{m}(1 \leq \mathrm{x} \leq 1.2) \rightarrow \mathrm{P} 21 / \mathrm{n}(1.4 \leq \mathrm{x} \leq 2.0)$. The substitution of $\mathrm{Ba} 2+$ for $\mathrm{Sr} 2+$ results in distortion of the double perovskite structure via increased tilting of the $\mathrm{BO} 6$ octahedra, due to the smaller ionic radius of $\mathrm{Sr} 2+$ relative to Ba2+. Magnetic ordering was determined for these structures using temperature dependent magnetic susceptibility, isothermal magnetisation measurements and low temperature neutron powder diffraction data. These compounds were found to be antiferromagnetic with two distinct ordering temperatures of the Ho3+ and Ru5+ ions, as indicated by two peaks in the susceptibility curves. The magnetic ordering temperature of the Ru5+ ions was found to decrease across the series with increasing Sr content. A transition from antiferromagnetic ordering to a canted antiferromagnetic ground state was indicated by magnetic hysteresis loops in isothermal magnetism measurements and correlates with the phase transition from $\mathrm{I} 2 / \mathrm{m}$ to $\mathrm{P} 21 / \mathrm{n}$ symmetry as a result of further distortion of the crystal structure. Divergence of ZFC and FC susceptibility curves also correlates with this phase transition, likely indicating the onset of magnetic frustration. A magnetic structure was refined for the end member Sr2HoRuO6 and indicates that both ruthenium and holmium cations cooperatively cant out of the AFM plane, leading to the magnetic ground state possessing a net ferromagnetic moment. Further research utilizing these techniques is being undertaken to elucidate crystal symmetrymagnetism correlations in mixed $4 \mathrm{~d}$ and $5 \mathrm{~d}$ double perovskites oxides.

[1] Battle, P. D. Macklin, W. J. (1984) J. Solid State Chem., 52 (2), 138-145.

[2] Wu, M. K. Chen, D. Y. Ling, D. C. Chien, F. Z. (2000) Physica B, 284, 477-478.

[3] Ranjbar, B. Pavan, A. Kennedy B. J. Zhang, Z. (2015) Dalton Trans., 44, 10689.

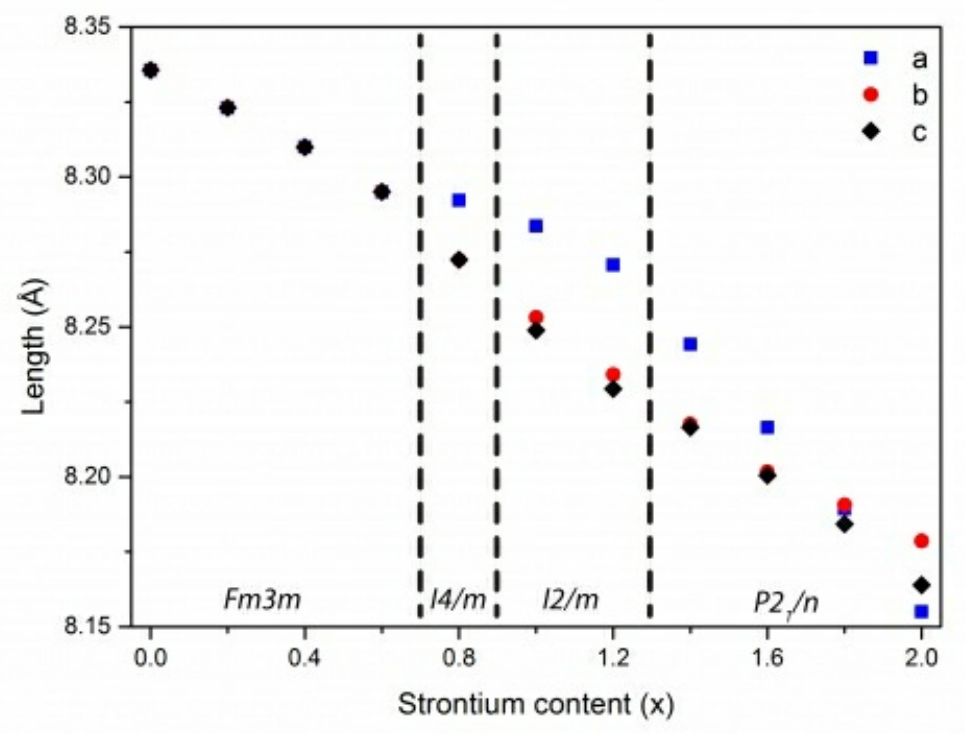

Keywords: Ruthenium Perovskite, Canted Antiferromagnetism 